\title{
Underlying mechanisms for syntrophic metabolism of essential enzyme cofactors in microbial communities
}

\author{
Margaret F Romine ${ }^{1}$, Dmitry A Rodionov ${ }^{2,3}$, Yukari Maezato ${ }^{1}$, Andrei L Osterman ${ }^{2}$ \\ and William C Nelson ${ }^{1}$ \\ ${ }^{1}$ Pacific Northwest National Laboratory, Richland, WA, USA; ${ }^{2}$ Sanford Burnham Prebys Medical Discovery \\ Institute, La Jolla, CA, USA and ${ }^{3}$ A.A. Kharkevich Institute for Information Transmission Problems, Russian \\ Academy of Sciences, Moscow, Russia
}

\begin{abstract}
Many microorganisms are unable to synthesize essential B vitamin-related enzyme cofactors de novo. The underlying mechanisms by which such microbes survive in multi-species communities are largely unknown. We previously reported the near-complete genome sequence of two $\sim 18$-member unicyanobacterial microbial consortia that maintain stable membership on defined medium lacking vitamins. Here we have used genome analysis and growth studies on isolates derived from the consortia to reconstruct pathways for biogenesis of eight essential cofactors and predict cofactor usage and precursor exchange in these communities. Our analyses revealed that all but the two Halomonas and cyanobacterial community members were auxotrophic for at least one cofactor. We also observed a mosaic distribution of salvage routes for a variety of cofactor precursors, including those produced by photolysis. Potentially bidirectional transporters were observed to be preferentially in prototrophs, suggesting a mechanism for controlled precursor release. Furthermore, we found that Halomonas sp. do not require cobalamin nor control its synthesis, supporting the hypothesis that they overproduce and export vitamins. Collectively, these observations suggest that the consortia rely on syntrophic metabolism of cofactors as a survival strategy for optimization of metabolic exchange within a shared pool of micronutrients.
\end{abstract}

The ISME Journal (2017) 11, 1434-1446; doi:10.1038/ismej.2017.2; published online 10 February 2017

\section{Introduction}

Recent improvements in sequencing technologies have made it possible to sequence entire communities and to use genome-enabled approaches for the study of microbial interactions that lead to community-level properties (Song et al., 2015). Because B vitamin-related enzyme cofactors are essential but expensive to produce, their precursors are commonly shared by community members. It is recognized that many microorganisms are unable to synthesize all required cofactors de novo and instead salvage select precursors to make them. The genetic basis for this salvage has been defined in many organisms, revealing a great diversity of transporters (Jaehme and Slotboom, 2015a), alternative enzymes and pathways for converting precursors to cofactors, and the tight regulation of precursor salvage and cofactor biosynthesis (Winkler et al., 2002a,b; Nahvi

Correspondence: WC Nelson, Pacific Northwest National Laboratory, 902 Battelle Boulevard, PO Box 999, MSIN J4-18, Richland, WA 99352, USA.

E-mail: William.Nelson@pnnl.gov

Received 31 May 2016; revised 11 November 2016; accepted 19 December 2016; published online 10 February 2017 et al., 2004; Ames et al., 2010; Leyn et al., 2016; Suvorova and Rodionov, 2016). New genes involved in cofactor metabolism continue to be discovered, largely due to the increasing availability of whole genome sequences and the ability of comparative genomics and regulon analysis to discover new protein families that participate in this process (Sun et al., 2013; Rodionova et al., 2015).

At present, studies of cofactor precursor exchange in natural communities have been limited to surveys of biosynthetic and salvage potential in metagenomes (Magnusdottir et al., 2015) where the sources of precursors are not defined, or in assays of binary cultures where the number of precursors exchanged are minimal (Croft et al., 2005; Kazamia et al., 2012; Grant et al., 2014). Here we focused instead on two nearly identical consortia derived from benthic microbial mats that occur in a heliothermal saline (epsomite, $\mathrm{MgSO}_{4}$ ) lake in northern Washington State (Lindemann et al., 2013; Zachara et al., 2016). These unicyanobacterial consortia (UCC) consist of a single, but distinct, cyanobacterium (Phormidesmis priestleyi ANA in UCC-A versus Phormidium sp. OSCR in UCC-O) and its associated heterotrophic cohorts (Cole et al., 2014) (Supplementary Table S1). 
Although the abundances of the heterotrophs relative to the cyanobacterium differ between the consortia, they each harbor the same 16 heterotrophs. A single additional heterotroph, Porphyrobacter sp. HL-46, was detected in only UCC-A (Nelson et al., 2016). Complete or near-complete genome sequences are available for most members enabling prediction of producers of cofactors and/or consumers of cofactor precursors. Importantly, no vitamin supplements were added to the growth medium during enrichment or subsequent cultivation. Hence, these consortia are dependent entirely on endogenous production of cofactors.

Here we used the subsystems approach and comparative genomics analysis to reconstruct vitamin cofactor biosynthetic pathways and predict transport capabilities in the 19 organisms that comprise these consortia. This approach, supported by supplementary growth studies on heterotrophic isolates, revealed an extensive cofactor auxotrophy among members of these consortia and differentiation in the type of precursors salvaged and the complement of cofactor-dependent pathways present. In addition, we identified potential transporters and regulators involved in precursor salvage. Insights gained from this study support the concept of syntrophic partnership based on vitamin exchange and suggest mechanisms by which this is achieved.

\section{Materials and methods}

Growth studies on UCC heterotrophic isolates

Hot Lake strains were routinely cultured at $30^{\circ} \mathrm{C}$ on Hot Lake Heterotroph broth, pH 8.0 (Cole et al., 2014). For testing vitamin-dependent growth, the cells were diluted to an $\mathrm{OD}_{600}$ of $\sim 0.2$ and inoculated into HL base medium (Hot Lake Heterotroph without yeast extract) supplemented with $5 \mathrm{~mm}$ carbon source and $1 \times$ Wolfe's vitamin mix (Wolin et al., $1963)$ or selected vitamins ( $0.74 \mathrm{~nm}$ cyanocobalamin, $0.015 \mu \mathrm{M}$ thiamine and/or $0.082 \mu \mathrm{M}$ biotin). When cultures reached mid-log phase, the cells were washed three times with HL base medium to remove residual metabolites and growth supplements. Ten replicate samples were evaluated every $1-2 \mathrm{~h}$ for all assays. For Erythrobacter HL-111, which grows slowly, the $\mathrm{OD}_{600}$ of replicated $20 \mathrm{ml}$ cultures was measured using a SmartSpec Plus Spectrophotometer (Bio-Rad, Hercules, CA, USA). For the remaining strains, a Norden Lab Professional-Bioscreen, $C$ Edition was used to analyze growth $\left(\mathrm{OD}_{600}\right)$ of $250 \mathrm{\mu l}$ samples in the 100-well Bioscreen plate.

\section{Consortial composition}

A combination of the species-resolved metagenome bins or isolate genome sequences (Nelson et al., 2016) from two unicyanobacterial consortia (UCC-A and UCC-O whose cyanobacterial members are designated Phormidium priestleyi ANA and Phormidesmis OSCR, respectively) were evaluated for the presence or absence of pathways for de novo biosynthesis of $\mathrm{B}$ vitamin-related enzyme cofactors. In addition to the cyanobacterial members, the closed genome sequences derived from 10 axenic cultures (Algoriphagus marincola str. HL-49, Aliidiomarina sp. HL-53, Roseibaca calidilacus HL-91, Halomonas sp. HL-48 and HL-93, Marinobacter excellens str. HL-55, Marinobacter sp. HL-58, Erythrobacter sp. HL-111, Salinivirga fredricksonii HL-109 and Porphyrobacter sp. HL-46) and binned assemblies from nine additional members (Bacteroidetes bin01, Oceanicaulis bin04 and Rhodobacteraceae bins 7, 8, 9, 12 and 18) were examined. Based on occurrence of conserved single-copy genes, it was estimated that the binned assemblies have a genomic coverage of at least $98 \%$ with the exception of one member, Rhodobacteraceae bin09, whose estimated coverage is $87.6 \%$ (Nelson et al., 2016).

\section{Genomic reconstruction of metabolic pathways and regulons}

Genome-based reconstruction of eight B vitaminrelated cofactor biosynthesis pathways in 19 UCC genomes was performed using the subsystem-based comparative genomic approach (Osterman et al., 2010) implemented in SEED/RAST (Aziz et al., 2008; Overbeek et al., 2014; Brettin et al., 2015) combined with genomic reconstruction of vitamin-specific transcriptional regulons and identification of candidate vitamin transporters as previously described (Rodionov et al., 2009; Rodionova et al., 2015). Extensive manual pathway curation was assisted by KEGG orthology assignments from the BlastKoala annotation tool (Kanehisa et al., 2016). Identification of orthologs in closely related genomes and their genome neighborhood analysis were performed in the IMG workspace (Markowitz et al., 2014). Cofactor requirements were asserted by cataloging respective enzymes from annotated genomes and connecting them to metabolic pathways in KEGG (Kanehisa et al., 2016) and SEED (Overbeek et al., 2014). Computational searches of $\mathrm{B}_{12}, \mathrm{FMN}$ and thiamine pyrophosphate (TPP) riboswitches were performed using covariance models from the Rfam database as recently reviewed in Sun and Rodionov (2014). The BirA, NrtR, NadQ regulons were reconstructed by the consistency check approach using the positional weight matrices of DNA motifs from the RegPrecise database as previously described (Leyn et al., 2016). A full list of reconstructed metabolic pathways and regulons is available in the Supplementary Data 1.

Genome sequence accession numbers

All sequence data were generated by the Joint Genome Institute. Assemblies derived from metagenome bins and from isolate genome sequence can 
be accessed via IMGer (https://img.jgi.doe.gov/cgibin/mer/main.cgi). In addition, binned metagenome assemblies have been deposited in the European Nucleotide Archive (ENA; www.ebi.ac.uk/ena) and Genbank under accession numbers LIHN00 000000 (Bacteroidetes Bin01), LJSH00000000 (Oceanicaulis Bin04), LJSU00000000 (Rhodobacterales Bin07), LJSF00000000 (Rhodobacterales Bin08), LJNT00000000 (Rhodobacterales Bin09), LJZR00 000000 ( $P$. priestleyi ANA), LJSV00000000 (Rhodobacterales Bin12), LJZT00000000 (Phormidum OSCR) and LJSY00000000 (Rhodobacterales Bin18). Isolate genomes have been deposited under accession numbers GCA_001458075.1 (Aliidiomarina sp. HL-53), JMLY00000000 (Marinobacter sp. HL-58), GCA_001517585.1 (R. calidilacus sp. HL-91), NZ_JA FX00000000 (A. marinocola sp. HL-49), JMMC000 00000 (Halomonas sp. HL-48), JYNR00000000 (M. excellens sp. HL-55), GCA_000744895.1 (Porphyrobacter sp. HL-46), GCA_001314745.1 (Halomonas sp. HL-93), GCA_001314785.1 (Salinivirga fredricksonii HL-109, formerly Rhizobiales sp. HL-109) and GCA_001314765.1 (Erythrobacter sp. HL-111).

\section{Results}

\section{Composition of UCC-A and UCC-O}

The unicyanobacterial consortia examined in this study are low complexity enrichments resulting from attempts to develop axenic cultures of cyanobacteria from mats found in Hot Lake Washington. The firm attachment of heterotrophs to the cyanobacteria have thus far made it impossible to isolate the autotroph, but resulted in communities that are expected to be enriched in interacting microbes because the heterotrophs cannot fix $\mathrm{CO}_{2}$ that was provided as the sole carbon source during enrichment. Metagenomic sequence was derived from two unicyanobacterial consortia (UCC-A and UCC-O), assembled and binned into 19-member sequences (Nelson et al., 2016). Because the two consortia were nearly identical in taxonomic composition (16 heterotrophs were common to both consortia), co-assembly of sequencing reads lead to near-complete assembly of nearly all members of the consortia (the abundance of a 20th Alphaproteobacterial member was too low to produce sufficient genomic coverage to be included in this study). Further improvement of member assemblies resulted from sequencing the genomes of axenic cultures of heterotrophs derived from the consortia. In addition to the six genomic sequences from heterotrophic members of the consortia (Porphyrobacter sp. HL-46, Halomonas sp. HL-48, Algoriphagus marincola HL-49, Aliidiomarinacea HL-53, Marinobacter excellens HL-55 and Marinobacter sp. HL-58) described previously, this study includes genomic sequence from four new UCC isolates, Roseibaca calidilacus HL-91, Halomonas sp. HL-93, Erythrobacter sp. HL-111 and
Salinivirga fredricksonii HL-109, bringing the total number of members with closed genomes to 10 . In summary, each community is composed of a single cyanobacterium belonging to the Oscillatoriales order, two Bacteroidetes, five Gammaproteobacteria and $\sim 10$ Alphaproteobacteria (see Supplementary Table S1).

Exchange of cofactor precursors is necessary to support essential metabolic processes

We used a subsystems-based genomic reconstruction approach to infer biosynthesis, recycling and salvage pathways for eight B vitamin-related enzyme cofactors in the 19 members of UCC-A and UCC-O. Our analysis revealed that all members are able to synthesize PLP (pyridoxal phosphate; Supplementary Figure S1) and flavin nucleotides (Supplementary Figure S2), but only the cyanobacteria and both Halomonas sp. can produce the remaining six cofactors (summarized in Table 1). Four of the Rhodobacteraceae (bins 7, 9, 12 and 18) belonging to the Roseobacter group are auxotrophic for six cofactors, and the fifth (bin08) is only able to synthesize CoA de novo, making this group the most dependent on cofactor exchange within the consortia. By contrast, the Gammaproteobacteria require precursors to produce no more than two cofactors, cobalamin and/or TPP, each.

The consortia consist of 10 TPP, 11 biotin and 13 cobalamin auxotrophs, making their precursors the most widely sought after. Analysis of cofactordependent processes confirmed that TPP is essential for central carbon metabolism of all species in the two consortia (Supplementary Data 1 and Figure 1). Critical TPP-dependent enzymes found in all members include transketolase, a pyruvate oxidase, a TCA enzyme that degrades 2-oxogluatrate and 1-deoxy-D-xylulose-5-phosphate synthase, which produces a key metabolite needed for thiamine, pyridoxal phosphate and terpenoid biosynthesis. A single biotin-dependent enzyme, acetyl-CoA carboxylase, which is essential for the initiation of fatty acid biosynthesis, is present in all isolates.

Cobalamin is required for essential processes such as methionine biosynthesis. However, it is not universally required due to mechanisms that bypass the need for it (dashed lines, Figure 1). Examples include the use of alternative enzymes (e.g., MetE to synthesize methionine) and pathways (e.g., methyl citrate pathway to degrade propionyl-CoA) that do not require cofactors or transporters that import metabolites that would otherwise require a cofactor for synthesis (e.g., MetT or MetNIQ for methionine). The Gammaproteobacterial members use the most cobalamin-independent pathways, which is surprising as the two Halomonas spp. are among five of the only members that can synthesize this metabolically expensive cofactor. Unlike S. fredricksonii HL-109, the Halomonas spp. do not control cobalamin synthesis with a riboswitch (Supplementary Data 2 
Table 1 Overview of predicted cofactor biosynthesis capability

\begin{tabular}{|c|c|c|c|c|c|c|c|c|c|}
\hline & \multirow[t]{2}{*}{ Est. completeness ${ }^{\mathrm{a}}$} & \multicolumn{8}{|c|}{$\begin{array}{c}\text { Associated vitamin group } \\
\text { Cofactor }\end{array}$} \\
\hline & & $\begin{array}{l}B_{1} \\
T P P\end{array}$ & $\begin{array}{l}B_{2} \\
F A D F M N\end{array}$ & $\begin{array}{l}B_{3} \\
N A D\end{array}$ & $\begin{array}{l}\mathrm{B}_{5} \\
\mathrm{CoA}\end{array}$ & $\begin{array}{l}B_{6} \\
P L P\end{array}$ & $\begin{array}{l}B_{7} \\
\text { Biotin }\end{array}$ & $\begin{array}{l}B_{9} \\
T H F\end{array}$ & $\begin{array}{l}B_{12} \\
\text { Cobinamide }\end{array}$ \\
\hline \multicolumn{10}{|l|}{ Cyanobacteria } \\
\hline Phormidesmis priestleyi ANA & $99 \%$ & Pro+ & Pro & Pro+ & Pro & Pro & Pro+ & Pro+ & Pro \\
\hline Phormidium OSCR & $99 \%$ & Pro & Pro & Pro+ & Pro & Pro & Pro+ & Pro+ & Pro+ \\
\hline \multicolumn{10}{|l|}{ Bacteroidetes } \\
\hline Bacteroidetes sp. bin01 & $\mathbf{9 9} \%$ & Aux & Pro & Pro & Pro+ & Pro & Pro+ & Pro & Aux \\
\hline Algoriphagus marincola HL-49 & $>99 \%$ & Aux & Pro & Pro+ & Pro & Pro & Aux & Pro & Aux \\
\hline \multicolumn{10}{|l|}{ Gammaproteobacteria } \\
\hline Aliidiomarina sp. HL-53 & $>99 \%$ & Aux & Pro & Pro & Pro+ & Pro & Pro+ & Pro & - \\
\hline Halomonas sp. HL-48 & $>99 \%$ & Pro+ & Pro & Pro+ & Pro+ & Pro & Pro+ & Pro & Pro+ \\
\hline Halomonas sp. HL-93 & $>99 \%$ & Pro+ & Pro & Pro+ & Pro+ & Pro & Pro+ & Pro & Pro+ \\
\hline Marinobacter excellens HL-55 & $>99 \%$ & Pro+ & Pro & Pro & Pro & Pro & Pro+ & Pro & Aux \\
\hline Marinobacter sp. HL-58 & $>99 \%$ & Pro+ & Pro & Pro+ & Pro & Pro & Pro+ & Pro & Aux \\
\hline \multicolumn{10}{|l|}{ Alphaproteobacteria } \\
\hline Erythrobacter sp. HL-111 & $>99 \%$ & Pro & Pro & Pro & Pro & Pro & $\operatorname{Aux}^{\mathbf{b}}$ & Pro & Aux \\
\hline Porphyrobacter sp. HL-46 & $>99 \%$ & Pro & Pro & Pro & Pro & Pro & $\operatorname{Aux}^{\mathrm{b}}$ & Pro & Aux \\
\hline Oceanicaulis bin04 & $99 \%$ & Pro & Pro & Pro & Pro & Pro+ & $\operatorname{Aux}^{\mathbf{b}}$ & Pro & Aux \\
\hline Salinivirga fredricksonii HL-109 & $>99 \%$ & Aux $^{\mathrm{c}}$ & Pro & Aux & Pro & Pro+ & Aux & Aux & Pro+ \\
\hline Roseibaca calidilacus HL-91 & $>99 \%$ & Aux & Pro & Pro+ & Pro & Pro & Aux & Pro & Aux \\
\hline Rhodobacteraceae bin12 & $>99 \%$ & Aux & Pro & Aux & Aux & Pro & Aux & Aux $^{d}$ & Aux \\
\hline Rhodobacteraceae bin07 & $>99 \%$ & Aux & Pro & Aux & Aux & Pro & Aux & Aux $^{d}$ & Aux \\
\hline Rhodobacteraceae bin08 & $>99 \%$ & Aux & Pro & Aux & Pro & Pro & Aux & Aux $^{d}$ & Aux \\
\hline Rhodobacteraceae bin09 & $\mathbf{8 8} \%$ & Aux & Pro & Aux & Aux & Pro & Aux & Aux $^{d}$ & Aux \\
\hline Rhodobacteraceae bin18 & $>\mathbf{9 9} \%$ & Aux & Pro & Aux & Aux & Pro+ & Aux & $\operatorname{Aux}^{d}$ & Aux \\
\hline
\end{tabular}

Abbreviations: Aux, auxotroph; CoA, coenzyme A; PLP, pyridoxal phosphate; Pro, prototroph; Pro+, prototroph with salvage capability; THF, tetrahydrofolate; TPP, thiamine pyrophosphate. Auxotrophies are indicated in bold.

aEstimated by presence/absence of 100 conserved single-copy genes (Nelson, et al., 2016). Organisms for which a sequenced isolate genome (improved draft) is available are listed as $>99 \%$. Metagenomic bins that contain all 100 marker genes are also listed as $>99 \%$.

${ }^{\mathrm{b}} \mathrm{Can}$ make biotin from dethiobiotin.

'Predicted to salvage HMP (hydroxymethylpyrimidine) and thiazole rather than thiamine.

${ }^{\mathrm{d}}$ Can make folate if provided pABA (para-aminobenzoic acid).

and Supplementary Note 1), and therefore have the potential to make excess cofactor and be important providers of $\mathrm{B}_{12}$ for the community.

\section{Exchange of canonical B vitamins can complement} most, but not all, deficiencies in de novo biosynthesis of respective cofactors

Although, by definition, B vitamins include a variety of different cofactor precursors (Supplementary Table S2), the chemicals included in the conventional medium supplement, Wolfe's vitamin solution (Wolin et al., 1963) are the standard growth supplements used to cultivate microbes and more widely assumed to be exchanged in microbial communities. Here we found, however, that pantothenate, nicotinic acid, folic acid, para-aminobenzoic acid (pABA), and cyanocobalamin are the only standard vitamin additives that can complement UCC member deficiencies in cofactor biosynthesis.

Pantothenate, a precursor to coenzyme A (CoA), is synthesized de novo through the actions of (i) PanB and PanE (or IlvC), which convert 3-methyl-2- oxobutanoate to pantoate and (ii) PanC, which ligates $\beta$-alanine to pantoate to form pantothenate (Figure 2). All UCC members can convert pantothenate to CoA, but four Rhodobacteraceae cannot synthesize pantothenate and thus are expected to be auxotrophs even though no pantothenate transporter could be identified. An aspartate carboxylase, PanD or PanP, that is needed for the synthesis of $\beta$-alanine from aspartate is present in all members except Phormidesmis ANA, R. calidilacus HL-91, Erythrobacter HL-111, Porphyobacter HL-46 and S. fredricksonii HL-109. As HL-91 and HL-111 can grow in defined medium lacking CoA precursors (Supplementary Figure S7), we hypothesize that an alternative aspartate carboxylase or novel pathway to synthesize $\beta$-alanine exists.

Six heterotrophs (S. fredricksonii HL-109, Rhodobacteraceae bins $7,8,9,12$ and 18) are unable to synthesize $\mathrm{NAD}(\mathrm{P})$ from aspartate or tryptophan (Supplementary Figure S3) and the same six cannot produce tetrahydrofolate (Supplementary Figure S4). The presence of the nicotinate phosphoribosyltransferase (PncB and PncB2) in all auxotrophs and six 


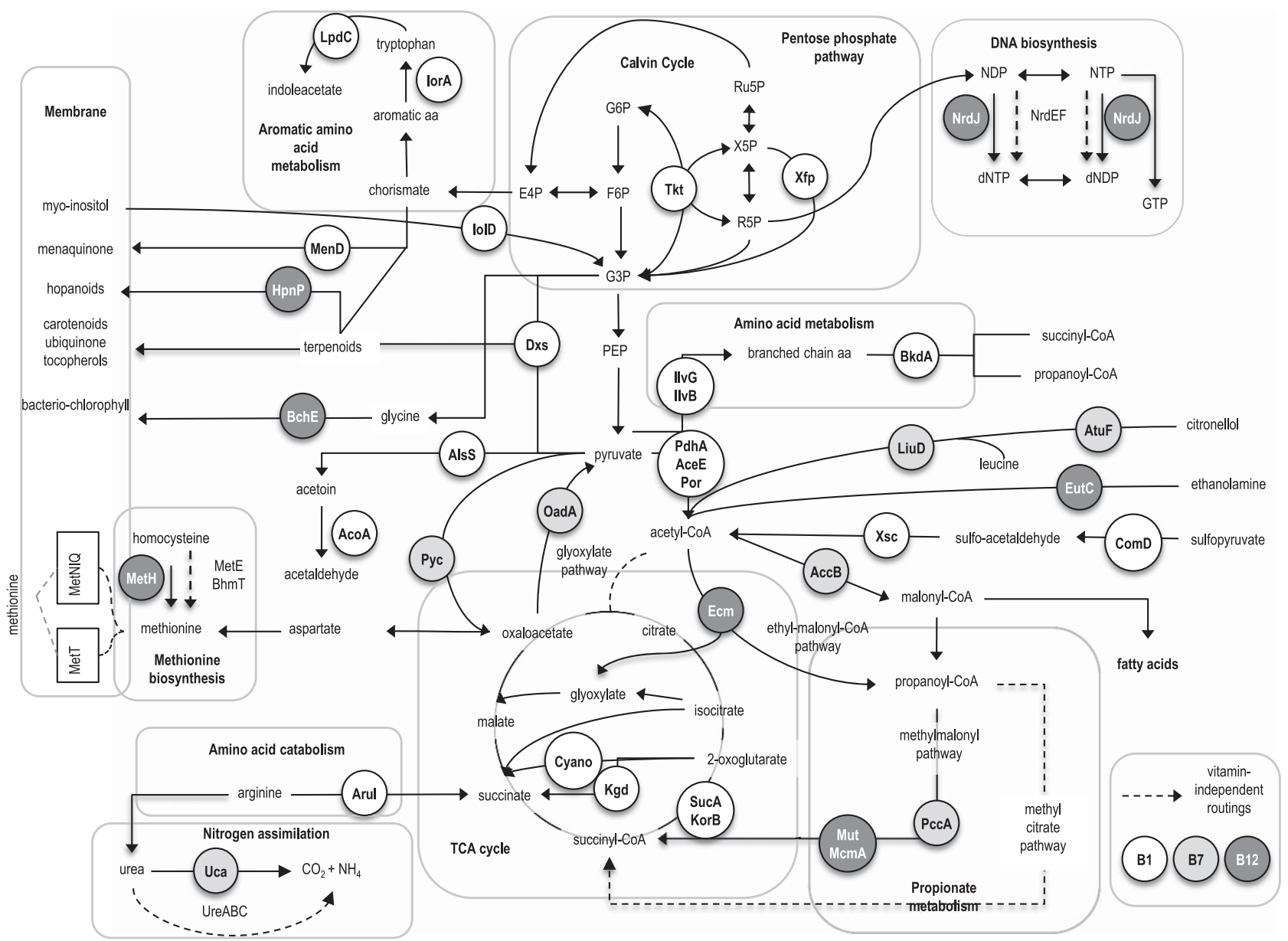

Figure 1 Overview of TPP, biotin and $\mathrm{B}_{12}$-dependent processes. TPP-dependent enzymes: AceE, pyruvate dehydrogenase E1 component; AcoA, acetoin dehydrogenase complex E1 component alpha subunit; AlsS, acetolactate synthase; AruI, 2-ketoarginine decarboxylase; BkdA, 2-oxoisovalerate dehydrogenase E1 component alpha subuit; ComD, sulfopyruvate decarboxylase alpha subunit; Cyano, 2oxoglutarate dehydrogenase; Dxs, 1-deoxy-d-xylulose-5-phosphate synthase; IlvB, biosynthetic acetolactate synthase I catalytic subunit; IlvG, biosynthetic acetolactate synthase II catalytic subunit; IolD, decyclizing 3D-(3,5/4)-trihydroxycyclohexane-1,2-dione acylhydrolase; IorA, ferredoxin oxidoreductase alpha subunit; Kgd, 2-oxoglutarate decarboxylase; KorB, 2-oxoglutarate ferredoxin oxidoreductase beta subunit; LpdC, indolepyruvate decarboxylase; MenD, 2-succinyl-5-enolpyruvyl-6-hydroxy-3-cyclohexene-1-carboxylic-acid synthase; SucA, 2-oxoglutarate dehydrogenase E1 component; Tkt, transketolase; Xfp, xylulose-5-phosphate/fructose-6-phosphate phosphoketolase; and Xsc, sulfoacetaldehyde acetyltransferase. Biotin-dependent enzymes: AccB, acetyl-CoA carboxylase biotin carboxyl carrier protein; AtuF, geranyl-CoA carboxylase alpha subunit; LiuD, 3-methylcrotonyl-CoA carboxylase alpha subunit; OadA, oxaloacetate decarboxylase alpha subunit; PccA, propionyl-CoA carboxylase alpha subunit; Pyc, pyruvate carboxylase; and Uca, urea carboxylase. $\mathrm{B}_{12}$-dependent enzymes: BchE, magnesium-protoporphyrin IX monomethyl ester cyclase; Ecm, (2R)-ethylmalonyl-CoA mutase; EutC, adenosylcobalamin-dependent ethanolamine ammonia-lyase small subunit; HpnP, hopanoid 2-methyltransferase; McmA, methylmalonyl-CoA mutase $\mathrm{B}_{12}$-binding subunit; MetH, methionine synthase; Mut, methylmalonyl-CoA mutase; and NrdJ, ribonucleoside diphosphate reductase. Enzymes and transporters that obviate the need for cofactors: BhmT, betaine-homocysteine methyltransferase; MetE, methionine synthase; MetNIQ, ABC-type methionine transporter; MetT, methionine:cation antiporter; NrdEF, ribonucleoside diphosphate reductase; UreABC, ABC-type urea transporter. Additional enzymes without precise functions predictions are listed in Supplementary Data 1 .

prototrophs suggest that nicotinic acid is salvaged, even though no candidate nicotinic acid transporters were identified. S. fredricksonii HL-109 is missing the entire pathway while five Rhodobacteraceae (bins 7, 8, 9, 12 and 18) lack only the ability to synthesize the precursor $p A B A$. These five members encode neither the enzymes with pABA synthetase or 4-amino-4-deoxychorismate lyase activity, which are needed to convert chorismate to $p A B A$ nor the recently described alternative pABA-biosynthetic enzyme (Adams et al., 2014; Satoh et al., 2014). No candidate folate or $p \mathrm{ABA}$ transporters were detected in respective auxotrophs, but four prototrophs (Phormidium OSCR, Oceanicaulis Bin04, Aliidiomarina HL-53 and Bacteroidetes Bin01) encode AbgT family transporters, which are proposed to export pABA (Delmar and $\mathrm{Yu}, 2016$ ), suggesting that they share this resource.

\section{A diverse set of precursors can support cobalamin auxotrophs}

Although cyanocobalamin is expected to support the growth of all the UCC cobalamin auxotrophs, the 


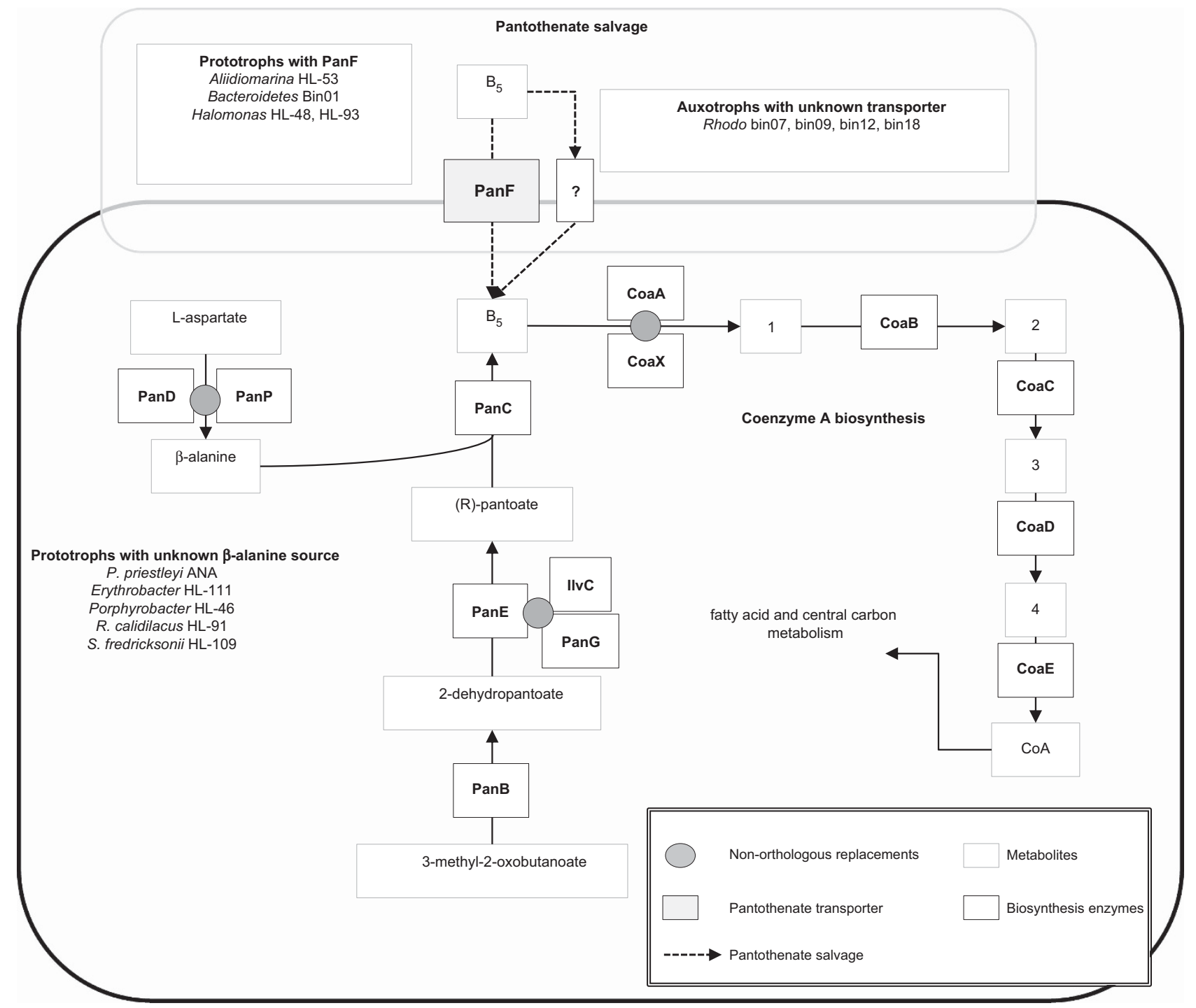

Figure 2 Coenzyme A biosynthesis and salvage of precursors. Metabolites that occur in the pathway for coenzyme A biosynthesis appear in open gray rectangles: 1, D-4'-phosphopantothenate; 2, (R)-4-phosophopantothenyoyl-L-cysteine; 3, pantetheine 4-phosphate; 4, dephospho-CoA; 5, N-carbomoyl- $\beta$-alanine; and CoA, coenzyme A. Enzymes appear in open black rectangles: CoaA, type I pantothenate kinase; CoaB, phosphopantothenate-cysteine ligase; CoaC, phosphopantothenoylcysteine decarboxylase; CoaD, pantetheine-phosphate adenylyltransferase; CoaE, dephospho-CoA kinase; CoaX, type III pantothenate kinase; IlvC, ketol-acid reductoisomerase; PanB, 3-methyl2-oxobutanoate hydroxymethyltransferase; PanC, pantoate- $\beta$-alanine ligase; PanD, pyruvoyl-dependent aspartate 1-decarboxylase; PanE, 2-dehydropantoate 2-reductase; PanG, ketopantoate reductase; PanP, PLP-dependent aspartate 1-decarboxylase; PreA, NAD-dependent dihydropyrimidine dehydrogenase subunit; PreT, NAD-dependent dihydropyrimidine dehydrogenase subunit; PydB, dihydropyriminidase; PydC, beta-ureidopropionase. PanF refers to the $\mathrm{Na}+$ /pantothenate symporter and the boxed question mark to unknown transporters. Non-orthologous replacements are indicated by filled gray circles. Dashed arrows indicate precursor salvage routes for coenzyme A precursor uptake.

presence of partial biosynthetic pathways in some members suggests that additional precursors might also be salvaged. The biosynthesis of $\mathrm{B}_{12}$ family cofactors is initiated by synthesis of the corrin ring from uroporphyrinogen III via either an oxygendependent (Warren et al., 2002; Heldt et al., 2005) or oxygen-sensitive pathway (Warren et al., 2002; Roessner and Scott, 2006; Supplementary Figure S5). Only five members (both cyanobacteria, both Halomonas spp. and S. fredricksonii HL-109) possess either of these pathways and hence synthesize cobalamin de novo. The second phase of $\mathrm{B}_{12}$ family cofactor biosynthesis involves attachment of the upper $\alpha$-axial component, which is derived from aminopropanol and either 5'-deoxyadensine or a methyl group, and a lower $\beta$-axial component typically composed of a cobalt-coordinated nucleotide with DMB (5,6-dimethylbenzimidazole) as the base (Gray and Escalante-Semerena, 2007; Supplementary Figure S6). The attachment of different combinations of ligands in the upper and lower positions results in the formation of distinct cofactors (e.g., pseudo- $\mathrm{B}_{12}$, methylcobalamin, adenosylcobalamin and hydroxocobalamine; Giedyk et al., 2015). 
1440

Both cyanobacteria genomes encode corrin ring biosynthetic genes, but lack cobT that is necessary for attachment of $\mathrm{DMB}$ as the lower ligand and, therefore, likely produce alternative coenzymes (Supplementary Figure S6) such as adenylylcobamide (pseudo- $\mathrm{B}_{12}$ ), which has been reported as the predominant form of $\mathrm{B}_{12}$ coenzyme in other cyanobacteria (Watanabe, 2007; Tanioka et al., 2009). Six auxotrophs (Bacteroidetes bin01, A. marincola HL-49, M. excellens HL-55, Porphyrobacter HL-46, Oceanicaulis bin04 and Rhodobacteraceae bin08) lack all biosynthetic genes and thus require an external source of cobalamin that cannot originate in the cyanobacteria. With the exception of Bacterioidetes bin01, the remaining auxotrophs encode partial pathways for biosynthesis of adenosylcobalamin, the TonB-dependent receptor (BtuB), and the ABCtype inner membrane translocase (BtuCDF), which are required for the uptake of cobalamin and related precursor corrinoids (Woodson et al., 2005; Degnan et al., 2014). The missing ABC transporter genes in Bacteroidetes bin01 are presumably in a sequencing gap. Interestingly, all three of the heterotrophic cobalamin producers lack the high affinity BtuB outer membrane receptor. Based on the gene complement present in these members, we predict that they salvage one or more of the precursors hydrogenobyrinic acid a,c-diamide, cobyrinic acid, cobyrate acid and cobinamide-guanosine diphosphate and consequently, some community members may benefit from more precursors than others (Figure 3).

\section{S. fredricksonii HL-109 and Rhodobacteraceae bino9} are TPP auxotrophs that cannot salvage thiamine The de novo biosynthesis of TPP involves the formation of phosphorylated hydroxyethylthiazole (HET) and hydroxymethylpyrimidine (HMP) intermediates, which are first joined by ThiE (thiamine-phosphate pyrophosphorylase) and then phosphorylated by ThiL (thiamine monophosphate kinase; Du et al., 2011; Figure 4). Only six members (each of the cyanobacteria, Halomonas spp. and Marinobacter spp.) have all the genes necessary for TPP biosynthesis. Erythrobacter HL-111, Porphyrobacter HL-46 and Oceanicaulis bin04 lack known enzymes for synthesis of the iminoglycine precursor. However, HL-111 can grow in defined medium lacking thiamine (Supplementary Figure S7), which suggests that an alternative iminoglycine biosynthesis pathway exists and that all three organisms are also prototrophs.

Two main salvage strategies are used by our consortia to produce TPP (Figure 5). Nine members of the consortia use the most commonly recognized strategy, which involves uptake of thiamine followed by its phosphorylation to TPP. Five different thiamine transporters were detected including ThiV2, which was discovered through our analysis (Supplementary Note 2). Five auxotrophs encode a partial TPP biosynthetic pathway (ThiD, ThiM, ThiE and ThiL), which can be used to convert salvaged HET and HMP into TPP. Two transporters were detected in auxotroph genomes; ThiXYZ that had been previously associated with HMP transport and a TRAP-type transporter that we predict to be used for HET uptake (Supplementary Note 2). The ThiV and/or TRAP transporters were also found in several TPP prototrophs, suggesting that they can reduce the cost of biosynthesis by salvaging HET and/or HMP. Salvage of both precursors is the only option for generating TPP by $S$. fredricksonii HL-109 and Rhodobacteraceae bin09 and thus Wolfe's vitamin solution should not support growth of these organisms.

Non-canonical vitamins can be salvaged for biotin, $N A D(P)$ and PLP biosynthesis

Over half of the community members are biotin auxotrophs (Table 1 and Supplementary Figure S8), but all are predicted to salvage biotin or a precursor. Biotin is salvaged by either the the YigM transporter or the BioY ECF-type transporter, which can function even in the absence of the auxiliary components BioMN (Finkenwirth et al., 2013). Three auxotrophs lack YigM and BioY but encode the biotin synthase (BioB), which catalyzes the last step in biosynthesis. Thus, we predict that dethiobiotin is a precursor that is available for exchange in communities and that transporters other than BioY or YigM mediate its uptake.

All six NAD(P) auxotrophs (S. fredricksonii HL-109, R. calidilacus HL-91 and Rhodobacteraceae bins 7, 8, 9, 12 and 18) and five of the prototrophs (both cynobacteria, A. marincola HL-49 and both Halomonas spp.) encode nicotinamidase (PncA) and, therefore, are expected to salvage nicotinamide in addition to nicotinic acid. A. marincola HL-49 encodes the PnuC transporter and NadR kinase, which is required for the salvage of nicotinamide riboside (Supplementary Figure S3). It is recognized that pyridoxine kinase (PdxK) can convert pyridoxine, pyridoxal or pyridoxamine into PLP and, therefore, the three prototrophs that have PdxK can potentially salvage any of these three precursors (Supplementary Figure S1).

\section{Biased distribution of one-component transporters among prototrophs and auxotrophs}

We observed a consistent trend in the presence of one-component transporters in cofactor prototroph genomes and not in auxotrophs, which suggests that prototrophs might conditionally export substrates (Figure 6, Supplementary Data 4). YigM, which was found in all six of the heterotrophic biotin prototrophs, has been demonstrated to take up biotin (Ringlstetter, 2010; Finkenwirth et al., 2013) yet is classified as a DME family transporter (TC: 2.A.7.3; Saier et al., 2014). Most members of this transporter family are implicated in export functions, supporting 


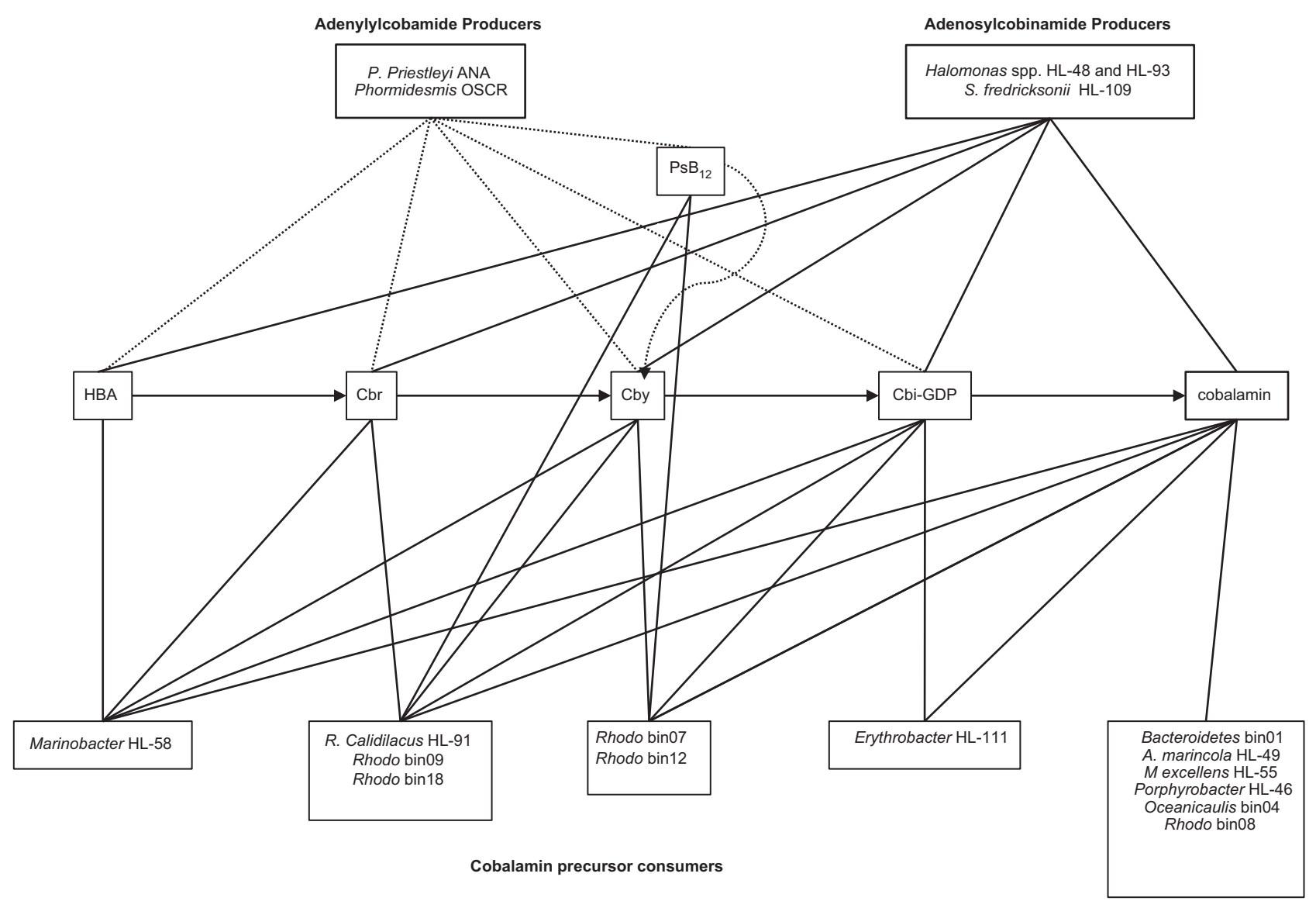

Figure 3 Model of potential cobalamin precursor exchange among UCC community members. The two types of $\mathrm{B}_{12}$ producers (adenylylcobamide versus adenosylcobinamide) are shown at the top and the auxotrophs are shown at the bottom. Intermediates that are predicted to be salvaged are shown in the middle; HBA, hydrogenobyrinic acid; Cbr, cobyrinate a,c-diamide; Cby, cobyrate; Cbi-GDP, cobinamide-guanosine diphosphate. Arrows between intermediates depict the order (earliest to latest from left to right) in which they enter the cobalamin biosynthetic pathway. Pseudo- $\mathrm{B}_{12}\left(\mathrm{PsB}_{12}\right)$ has an adenine rather than a 5,6-dimethylbenzimidazole (DMB) at the alpha-axial ligand position. Note that auxotrophs at the far bottom right are unable to make use of precursors produced by the cyanobacteria.

the notion that this transporter operates in both directions.

Four transporters are classified as $\mathrm{Na}^{+}$or $\mathrm{H}^{+}$:solute symporters and thus translocation directionality is likely controlled by $\mathrm{pH}$ and ionic strength. Three transporters (ThiV, ThiV2 and PanF) belong to the $\mathrm{Na}^{+}$:solute symporter family (TC: $2 . \mathrm{A} .21$ ) and the fourth, Fbt, belongs to the folate-biopterin transporter family (TC: 2.A.71). Among these four, ThiV2 is the only one not found exclusively in prototrophs. Two additional one-component transporters, PnuC and PnuT, catalyze transport by facilitated diffusion. Their substrates are phosphorylated by a kinase after uptake, thereby trapping them inside the cell (Jaehme and Slotboom, 2015b). Transport directionality control would, therefore, be impacted by the energy status of the host. PnuC is found in the NAD prototroph, A. marincola HL-49, and PnuT is found in a single auxotroph, Aliidiomarina sp. HL-53. Taking into account other microorganisms that encode PnuC and PnuT, it is evident that their distribution is uneven, but most occur in prototrophs.

\section{Discussion}

Our results demonstrate, for the first time, how a selfsustaining defined microbial community can retain members that have numerous essential requirements for B vitamin-related enzyme cofactor precursors (Figure 6). The analysis of member biosynthetic capabilities revealed that many of the auxotrophs encode partial biosynthetic pathways that initiate with metabolites that are precursors of canonical vitamins rather than the vitamins themselves. Diversification and specialization of precursor salvage provides a mechanism for division of labor in microbial communities and a selective advantage for partners uniquely suited for optimal syntrophic biosynthesis of cofactors (see Fredrickson, 2015). These findings also have important implications for the cultivation of microbes from environmental samples, where the naive assumption is that the metabolites in Wolfe's supplement are sufficient to support cofactor biosynthesis deficiency or to enable a desired community member to outcompete its neighbors. Although alternate salvage systems have 


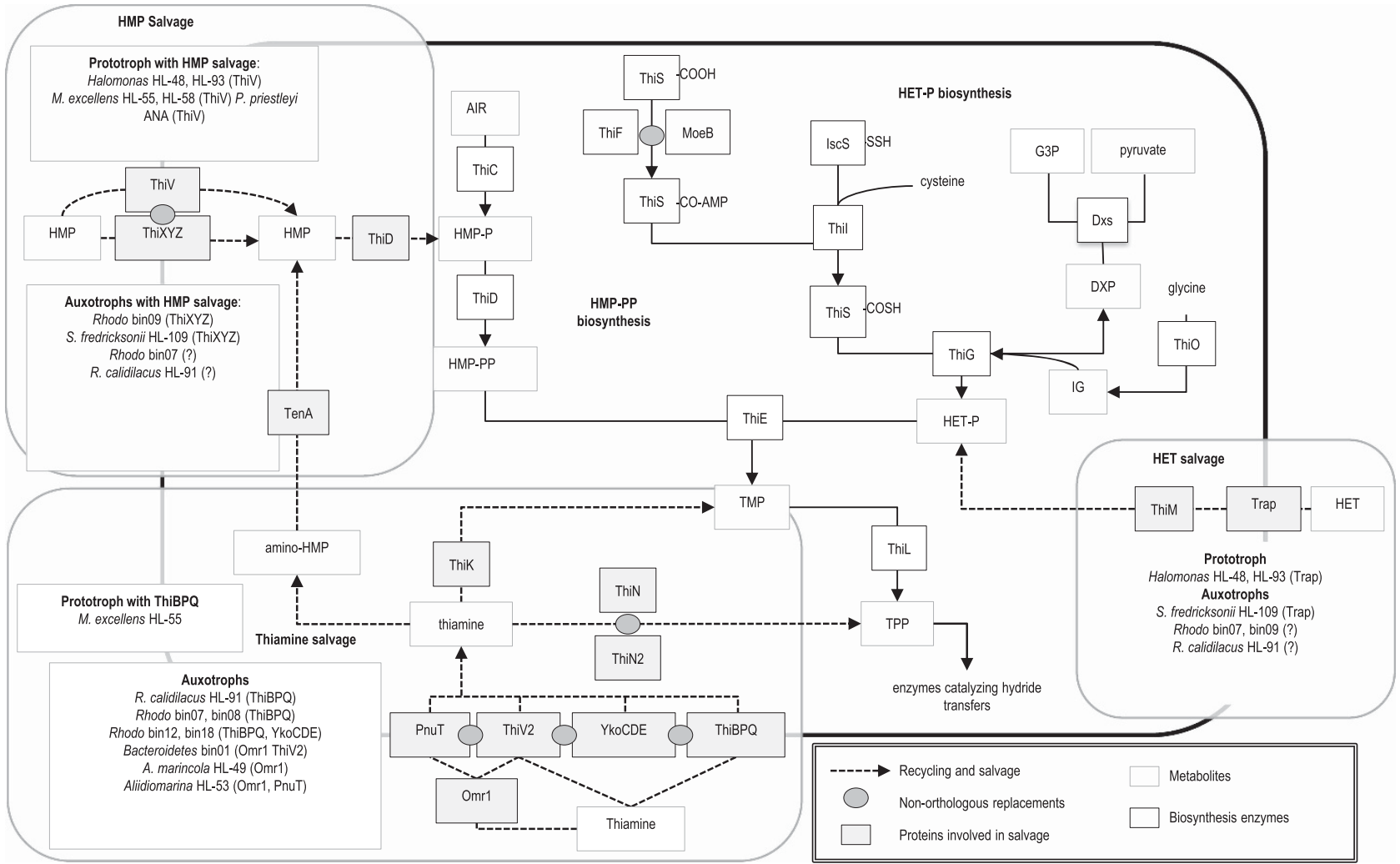

Figure 4 Thiamine, hydroxyethylthiazole and 4-amino-t-aminomethyl-2-methylpyrimidine are salvaged for thiamine pyrophosphate biosynthesis. Metabolites that occur in the thiamine pyrophosphate biosynthetic and precursor salvage pathways: AIR, 5-amino-1-(5phospho-D-ribosyl)imidazole; amino-HMP, 4-amino-5-aminomethyl-2-methylpyrimidine; DXP, 1-deoxy-D-xylulose-5-phosphate; G3P, glyceraldehyde-3-phosphate; HET, hydroxyethylthiazole; HMP, 4-amino-5-hydroxymethyl-2-methylpyrimidine; IG, iminoglycine; -P, phosphate; -PP, diphosphate; TMP, thiamine monophosphate; TPP, thiamine pyrophosphate. Enzymes involved in biosynthesis: CdsH, cysteine desulfhydrase; Dxs, 1-deoxy-d-xylulose-5-phosphate synthase; IscS, cysteine desulfurase; MoeB, adenylyltransferase/sulfurtransferase; ThiC, phosphomethylpyrimidine synthase; ThiD, bifunctional hydroxymethylpyrimidine/phosphomethylpyrimidine kinase; ThiE, thiamine-phosphate pyrophosphorylase; ThiF, sulfur carrier protein adenylyltransferase; ThiG, thiazole synthase; ThiL, thiamine monophosphate kinase; ThiO, glycine oxidase; and ThiS, sulfur carrier protein. Enzymes involved in salvage and recycling: TenA, thaiminase; ThiK, thiamine kinase; ThiM, 4-methyl-5-(beta-hydroxyethyl) thiazole kinase; ThiN and ThiN2, thiamine pyrophosphokinase. Thiamine transporters include the Omr1 TonB-dependent receptor, the PnuT ATP-dependent transporter, the ThiBPQ ABC-type transporter, The YkoCDE ECF-type transporter and the ThiV2 sodium-dependent transporter. Hydroxymethylpyrimidine transporters are the ThiV sodium-dependent transporter and the ThiXYZ ABC-type transporter. Trap refers to the single unnamed Trap-type hydroyethylthiazole transporter.

been described previously, (Taga and Walker, 2008; Galeazzi et al., 2011), this knowledge has yet to be broadly applied to microbial physiology and cultivation. When cultivating uncharacterized microbes, we advise supplementing the standard vitamin supplements with additional precursors such as those described herein.

Precursor specialization may be driven by environmental conditions affecting precursor availability. Our consortia are grown under constant light conditions, and two cofactors, TPP and adenosylcobalamin, are susceptible to photolysis. Photolysis of thiamine yields HMP and HET (Ansari et al., 2004). Six of the community members have salvage systems for HMP and HET, with four members restricted to salvage of these precursors rather than thiamine itself (Figure 4 and Supplementary Data 3). Exclusive use of these metabolites likely reflects their ready availability. Photolysis of adenosylcobalamin causes loss of its adenosyl group (Garabato et al., 2016).
Although it is predicted that all community members can salvage cob(II)alamin, it is likely that they do not all perform this task with the same efficiency. We predict that, in situ, concentrations of TPP and cobalamin precursors vary over the diel cycle and across mat depth, and that this variation may drive shifts in community function or composition.

Two mechanisms proposed for the release of vitamins to consumers are passive exchange, in which death and lysis of the producers benefits the consumers (Karl, 2002), and direct symbiosis, where producers synthesize excess vitamin and export it for use by consumers. Although passive exchange is assumed to occur in all systems, mathematical modeling of the growth dynamics of an obligate syntrophic partnership between a bacterial $B_{12}$ producer and an algal consumer has provided strong evidence for direct symbiosis (Grant et al., 2014). Our data suggest that direct symbiosis occurs in this community. The predominant member in each of our 


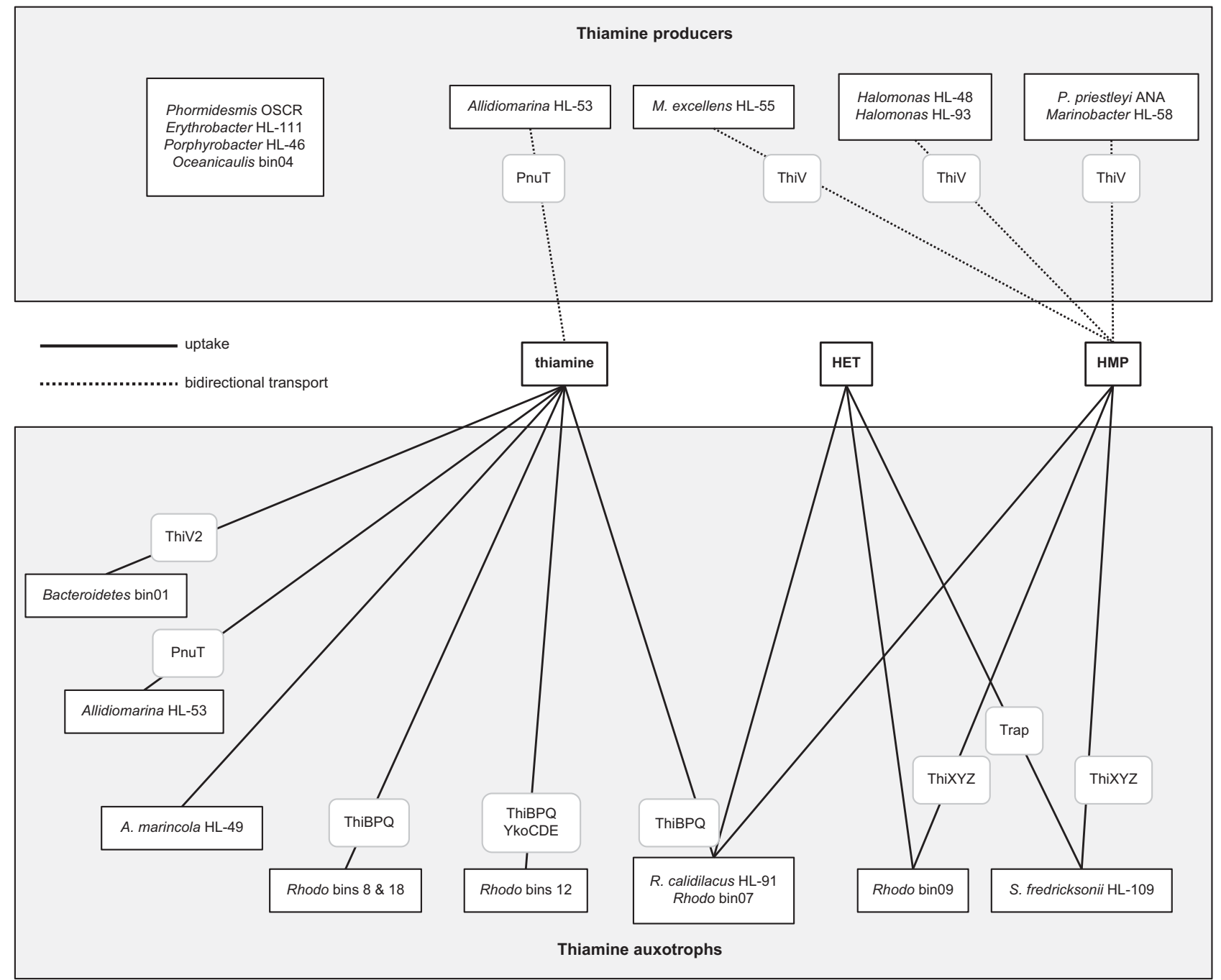

Figure 5 Overview of proposed interactions between thiamine producers and auxotrophs. TPP precursor transporters are depicted by rounded rectangles with gray edges. Precursors that are exchanged include thiamine, hydroxyethylthiazole (HET) and 4-amino-5hydroxymethyl-2-methylpyrimidine (HMP). An exporter for HET has not been identified. Note that M. excellens HL-55 and both Halomonas spp. also have uptake transporters, but they are not shown here for clarity.

consortia is the cyanobacterium (P. priestleyi ANA or Phormidium OSCAR), which is prototrophic for all vitamins (Cole et al., 2014). However, the cyanobacteria are unable to produce adenosylcobalamin, which is required by six members of our community (Figure 3). Consequently, these six members must acquire cobalamin from the low-abundance producers, Halomonas HL-48 and HL-93 and S. fredricksonii HL-109. For these members to provide the needed precursors through lysis, a high turnover of cells would be necessary. In addition, we observed a preferential occurrence of energy-independent vitamin transporters of biotin, precursors to TPP, CoA, folate and NAD in respective vitamin producers in our community. We propose that these transporters are conditionally bidirectional, a hypothesis that is supported by the identification of the RibM energyindependent facilitator (transporter family 4.B.1) as a likely bidirectional translocator of riboflavin in Streptomyces davawensis (Hemberger et al., 2011).
Orthologs to RibM (designated PnuX) are found in nearly 400 genomes in the SEED database (see 'Riboflavin biosynthesis and transport' subsystem in http://pubseed.theseed.org/SubsysEditor.cgi), with only 10 predicted to lack riboflavin biosynthetic genes. The occurrence of energy-independent vitamin transporters is widespread among microbes. If many are indeed bidirectional, it suggests that syntrophic partnerships that rely on them are important in shaping community behavior and structure.

Although $\mathrm{B}_{12}$ is assumed to be exchanged between prototrophs and auxotrophs, no $\mathrm{B}_{12}$ exporters were identified. We speculate that one exists because previous studies suggest that Halomonas forms a syntrophic partnership with algae, with the former providing $\mathrm{B}_{12}$ and the latter a source of carbon (Croft et al., 2005). The Halomonas spp. in our community are the only members besides $S$. fredricksonii HL-109 that can provide suitable forms of cobalamin 


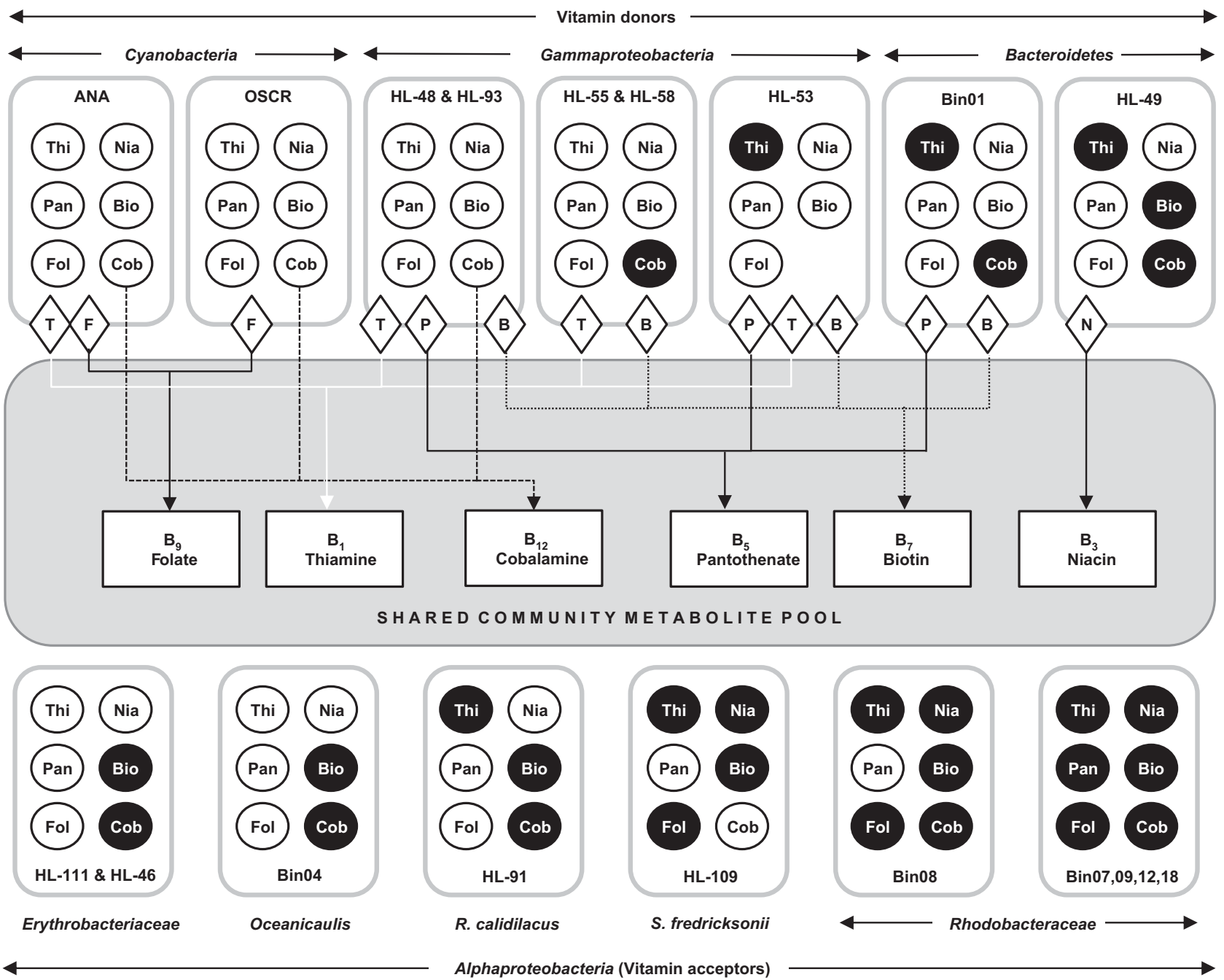

Figure 6 Reconstruction of syntrophic metabolism of B vitamin-related cofactors. Cofactor prototrophy and auxotrophy is represented by white and black ovals, respectively. Diamonds represent proposed bidirectional transporters $(\mathrm{T}=\mathrm{PnuT}$ in HL-53 and ThiV in the rest, $\mathrm{B}$ : YigM, F: Fbt, P: PanF, N: PnuC) of cofactors or their precursors. Text within ovals and diamonds refers to precursor groups (vitamins) that contribute to the community metabolite pool: thiamine: Thi or T; niacin: Nia or N; pantothenate: Pan or P; biotin: Bio or B; folate: Fol or F; cobalamin: Cob. No cobalamin information is shown for Aliidiomarina HL-53 because it encodes no cobalamin-dependent enzymes. The only required precursor with no candidate exporter is cobalamin, but we speculate that one exists.

for all the members. Furthermore, unlike HL-109, their biosynthetic genes are not under control of the $B_{12}$ riboswitch, and they encode enzymes or pathways that bypass essential requirements for this cofactor. Therefore, biosynthesis of cobalamin destined for export has reasonable potential to be achievable.

Metabolic reconstruction of cofactor biosynthesis and salvage pathways predicted the presence of precursor transporters (importers) that we were unable to identify. The most notable absence is a nicotinamide or nicotinate transporter required by 13 organisms in our system. A gene with this function has also been elusive in Escherichia coli; no candidate nicotinate transporter has been identified in Escherichia coli even though one has been known to exist for over 30 years (Rowe et al., 1985). One possibility is that a dedicated transporter does not exist and that available levels of precursor are sufficiently high to allow for passive transport. Other systems for which we could not identify transporters (folate, pantothenate and biotin) lack recognizable vitamin-responsive regulators, hampering efforts to use regulon analysis to identify these and other system components.

\section{Conclusion}

Our genomics-based metabolic reconstruction revealed that most members of the consortia have an essential requirement for cofactor precursors and thus must obtain them from other members of the community. We also found that some of the microbes have the potential to salvage a broader variety of precursors than are currently routinely used as growth supplements. Besides impacting strategies for the cultivation of microbes, this knowledge 
suggests that an organizational principle of metabolite exchange in communities is to diversify utilization of precursors. Last, our analyses revealed that cofactor producers typically encode different classes of transporters than do cofactor auxotrophs. We hypothesize that transporters translocate cofactor precursors inward or outward depending on the metabolic status of the cell, which suggests that the realized demand for precursors is dynamic, and that by balancing metabolite production and usage, a community can reap the benefits of division of labor. Therefore, while cell lysis could contribute to the shared pool of nutrients, the availability of cofactor precursors in the pool can also be controlled by the metabolic status and export capabilities of respective prototrophs. Furthermore, because certain processes that depend on cofactors are essential, they can serve as control points for coordination of community member abundance and function, suggesting that division of labor in cofactor production is closely tied to community emergent properties. Important knowledge gaps that remain to be explored include the characterization of mechanisms by which cofactor precursors are made available to the community and the means by which levels of precursor pools are controlled.

\section{Conflict of Interest}

The authors declare no conflict of interest.

\section{Acknowledgements}

This work was supported by the U.S. Department of Energy (DOE) Genome Sciences Program (GSP), Office of Biological and Environmental Research (OBER) and is a contribution of the Pacific Northwest National Laboratory (PNNL) Foundational Scientific Focus Area. Sequencing was done at the DOE Joint Genome Institute under contract no. DEAC02-05CH11231 and Community Science Project 701. Additional funding for work conducted by Dr Rodionov was provided by the Russian Science Foundation (14-1400289).

\section{References}

Adams NE, Thiaville JJ, Proestos J, Juarez-Vazquez AL, McCoy AJ, Barona-Gomez F et al. (2014). Promiscuous and adaptable enzymes fill 'holes' in the tetrahydrofolate pathway in Chlamydia species. MBio 5: e01378-01314.

Ames TD, Rodionov DA, Weinberg Z, Breaker RR. (2010). A eubacterial riboswitch class that senses the coenzyme tetrahydrofolate. Chem Biol 17: 681-685.

Ansari IA, Vaid FH, Ahmad I. (2004). Chromatographic study of photolysis of aqueous cyanocobalamin solution in presence of vitamins B and C. Pak J Pharm Sci 17: 19-24.

Aziz RK, Bartels D, Best AA, DeJongh M, Disz T, Edwards RA et al. (2008). The RAST Server: rapid annotations using subsystems technology. BMC Genomics 9: 75.
Brettin T, Davis JJ, Disz T, Edwards RA, Gerdes S, Olsen GJ et al. (2015). RASTtk: a modular and extensible implementation of the RAST algorithm for building custom annotation pipelines and annotating batches of genomes. Sci Rep 5: 8365.

Cole JK, Hutchison JR, Renslow RS, Kim YM, Chrisler WB, Engelmann HE et al. (2014). Phototrophic biofilm assembly in microbial-mat-derived unicyanobacterial consortia: model systems for the study of autotrophheterotroph interactions. Front Microbiol 5: 109.

Croft MT, Lawrence AD, Raux-Deery E, Warren MJ, Smith AG. (2005). Algae acquire vitamin $\mathrm{B}_{12}$ through a symbiotic relationship with bacteria. Nature 438: 90-93.

Degnan PH, Barry NA, Mok KC, Taga ME, Goodman AL. (2014). Human gut microbes use multiple transporters to distinguish vitamin $\mathrm{B}_{12}$ analogs and compete in the gut. Cell Host Microbe 15: 47-57.

Delmar JA, Yu EW. (2016). The AbgT family: a novel class of antimetabolite transporters. Protein Sci 25: 322-337.

$\mathrm{Du}$ Q, Wang H, Xie J. (2011). Thiamin (vitamin $\mathrm{B}_{1}$ ) biosynthesis and regulation: a rich source of antimicrobial drug targets? Int J Biol Sci 7: 41-52.

Finkenwirth F, Kirsch F, Eitinger T. (2013). Solitary BioY proteins mediate biotin transport into recombinant Escherichia coli. J Bacteriol 195: 4105-4111.

Fredrickson JK. (2015). ECOLOGY. Ecological communities by design. Science 348: 1425-1427.

Galeazzi L, Bocci P, Amici A, Brunetti L, Ruggieri S, Romine $\mathrm{M}$ et al. (2011). Identification of nicotinamide mononucleotide deamidase of the bacterial pyridine nucleotide cycle reveals a novel broadly conserved amidohydrolase family. J Biol Chem 286: 40365-40375.

Garabato BD, Lodowski P, Jaworska M, Kozlowski PM. (2016). Mechanism of Co-C photodissociation in adenosylcobalamin. Phys Chem Chem Phys 18: 19070-19082.

Giedyk M, Goliszewska K, Gryko D. (2015). Vitamin $B_{12}$ catalysed reactions. Chem Soc Rev 44: 3391-3404.

Grant MA, Kazamia E, Cicuta P, Smith AG. (2014). Direct exchange of vitamin $B_{12}$ is demonstrated by modelling the growth dynamics of algal-bacterial cocultures. ISME J 8: 1418-1427.

Gray MJ, Escalante-Semerena JC. (2007). Single-enzyme conversion of $\mathrm{FMNH}_{2}$ to 5,6-dimethylbenzimidazole, the lower ligand of $\mathrm{B}_{12}$. Proc Natl Acad Sci USA 104: 2921-2926.

Heldt D, Lawrence AD, Lindenmeyer M, Deery E, Heathcote P, Rigby SE et al. (2005). Aerobic synthesis of vitamin $\mathrm{B}_{12}$ : ring contraction and cobalt chelation. Biochem Soc Trans 33: 815-819.

Hemberger S, Pedrolli DB, Stolz J, Vogl C, Lehmann M, Mack M. (2011). RibM from Streptomyces davawensis is a riboflavin/roseoflavin transporter and may be useful for the optimization of riboflavin production strains. BMC Biotechnol 11: 119.

Jaehme M, Slotboom DJ. (2015a). Diversity of membrane transport proteins for vitamins in bacteria and archaea. Biochim Biophys Acta 1850: 565-576.

Jaehme M, Slotboom DJ. (2015b). Structure, function, evolution, and application of bacterial Pnu-type vitamin transporters. Biol Chem 396: 955-966.

Kanehisa M, Sato Y, Morishima K. (2016). BlastKOALA and GhostKOALA: KEGG tools for functional characterization of genome and metagenome sequences. J Mol Biol 428: 726-731. 
Karl DM. (2002). Nutrient dynamics in the deep blue sea Trends Microbiol 10: 410-418.

Kazamia E, Czesnick H, Nguyen TT, Croft MT, Sherwood E, Sasso $\mathrm{S}$ et al. (2012). Mutualistic interactions between vitamin $\mathrm{B}_{12}$-dependent algae and heterotrophic bacteria exhibit regulation. Environ Microbiol 14: 1466-1476.

Leyn SA, Stepanova VV, Suvorova IA, Kazakov AE, Novichkov PS, Rodionov DA et al. (2016). Comparative genomics and evolution of transcriptional regulons in Proteobacteria. Microb Genomics; e-pub ahead of print 7 July 2016; doi:10.1099/mgen.0.000061.

Lindemann SR, Moran JJ, Stegen JC, Renslow RS, Hutchison JR, Cole JK et al. (2013). The epsomitic phototrophic microbial mat of Hot Lake, Washington: community structural responses to seasonal cycling. Front Microbiol 4: 323.

Magnusdottir S, Ravcheev D, de Crecy-Lagard V, Thiele I. (2015). Systematic genome assessment of B-vitamin biosynthesis suggests co-operation among gut microbes. Front Genet 6: 148.

Markowitz VM, Chen IM, Palaniappan K, Chu K, Szeto E, Pillay M et al. (2014). IMG 4 version of the integrated microbial genomes comparative analysis system. Nucleic Acids Res 42: D560-D567.

Nahvi A, Barrick JE, Breaker RR. (2004). Coenzyme $B_{12}$ riboswitches are widespread genetic control elements in prokaryotes. Nucleic Acids Res 32: 143-150.

Nelson WC, Maezato Y, Wu YW, Romine MF, Lindemann SR. (2016). Identification and resolution of microdiversity through metagenomic sequencing of parallel consortia. Appl Environ Microbiol 82: 255-267.

Osterman AL, Overbeek R, Rodionov DA. (2010). The use of subsystems to encode biosynthesis of vitamins and cofactors. In: Mander LN, Liu H (eds). Comprehensive Natural Products II: Chemistry and Biology. Elsevier Ltd.: Kidlington, UK, pp 141-159.

Overbeek R, Olson R, Pusch GD, Olsen GJ, Davis JJ, Disz T et al. (2014). The SEED and the Rapid Annotation of microbial genomes using Subsystems Technology (RAST). Nucleic Acids Res 42: D206-D214.

Ringlstetter SL. (2010). Identification of the biotin transporter in Escherichia coli, biotinylation of histones in Saccharomyces cerevisiae and analysis of biotin sensing in Saccharomyces cerevisiae. $\mathrm{PhD}$ thesis, University of Regensburg, Regensburg, Germany.

Rodionov DA, Hebbeln P, Eudes A, ter Beek J, Rodionova IA, Erkens GB et al. (2009). A novel class of modular transporters for vitamins in prokaryotes. J Bacteriol 191: $42-51$.

Rodionova IA, Li X, Plymale AE, Motamedchaboki K, Konopka AE, Romine MF et al. (2015). Genomic distribution of B-vitamin auxotrophy and uptake transporters in environmental bacteria from the Chloroflexi phylum. Environ Microbiol Rep 7: 204-210.
Roessner CA, Scott AI. (2006). Fine-tuning our knowledge of the anaerobic route to cobalamin (vitamin $\mathrm{B}_{12}$ ). $J$ Bacteriol 188: 7331-7334.

Rowe JJ, Lemmon RD, Tritz GJ. (1985). Nicotinic acid transport in Escherichia coli. Microbios 44: 169-184.

Saier MH Jr, Reddy VS, Tamang DG, Vastermark A. (2014). The transporter classification database. Nucleic Acids Res 42: D251-D258.

Satoh Y, Kuratsu M, Kobayashi D, Dairi T. (2014). New gene responsible for para-aminobenzoate biosynthesis. J Biosci Bioeng 117: 178-183.

Song HS, Renslow RS, Fredrickson JK, Lindemann SR. (2015). Integrating ecological and engineering concepts of resilience in microbial communities. Front Microbiol 6: 1298.

Sun EI, Leyn SA, Kazanov MD, Saier MH Jr, Novichkov PS, Rodionov DA. (2013). Comparative genomics of metabolic capacities of regulons controlled by cis-regulatory RNA motifs in bacteria. BMC Genomics 14: 597.

Sun EI, Rodionov DA. (2014). Computational analysis of riboswitch-based regulation. Biochim Biophys Acta 1839: 900-907.

Suvorova IA, Rodionov DA. (2016). Comparative genomics of pyridoxal 5'-phosphate-dependent transcription factor regulons in Bacteria. Microb Genomics; e-pub ahead of print 19 January 2016; doi:10.1099/ mgen.0.000047.

Taga ME, Walker GC. (2008). Pseudo-B12 joins the cofactor family. J Bacteriol 190: 1157-1159.

Tanioka Y, Yabuta Y, Yamaji R, Shigeoka S, Nakano Y, Watanabe F et al. (2009). Occurrence of pseudovitamin $\mathrm{B}_{12}$ and its possible function as the cofactor of cobalamin-dependent methionine synthase in a cyanobacterium Synechocystis sp. PCC6803. J Nutr Sci Vitaminol (Tokyo) 55: 518-521.

Warren MJ, Raux E, Schubert HL, Escalante-Semerena JC. (2002). The biosynthesis of adenosylcobalamin (vitamin $\mathrm{B}_{12}$ ). Nat Prod Rep 19: 390-412.

Watanabe F. (2007). Vitamin $B_{12}$ sources and bioavailability. Exp Biol Med (Maywood) 232: 1266-1274.

Winkler W, Nahvi A, Breaker RR. (2002a). Thiamine derivatives bind messenger RNAs directly to regulate bacterial gene expression. Nature 419: 952-956.

Winkler WC, Cohen-Chalamish S, Breaker RR. (2002b). An mRNA structure that controls gene expression by binding FMN. Proc Natl Acad Sci USA 99: 15908-15913.

Wolin EA, Wolin MJ, Wolfe RS. (1963). Formation of methane by bacterial extracts. J Biol Chem 238: 2882-2886.

Woodson JD, Reynolds AA, Escalante-Semerena JC. (2005). ABC transporter for corrinoids in Halobacterium sp. strain NRC-1. J Bacteriol 187: 5901-5909.

Zachara JM, Moran JJ, Resch CT, Lindemann SR, Felmy AR, Bowden ME et al. (2016). Geo- and biogeochemical processes in a heliothermal hypersaline lake. Geochim Cosmochim Acta 181: 144-163.

Supplementary Information accompanies this paper on The ISME Journal website (http://www.nature.com/ismej) 